\title{
A NON-COMMUTATIVE SEMI-DISCRETE TODA EQUATION AND ITS QUASI-DETERMINANT SOLUTIONS
}

\author{
C. X. LI \\ School of Mathematical Sciences, Capital Normal University, Beijing 100048, China \\ e-mail: trisha_li2001@yahoo.com \\ and J. J. C. NIMMO \\ Department of Mathematics, University of Glasgow, Glasgow G12 8QW, UK \\ e-mail:j.nimmo@maths.gla.ac.uk
}

\begin{abstract}
A non-commutative version of the semi-discrete Toda equation is considered. A Lax pair and its Darboux transformations and binary Darboux transformations are found and they are used to construct two families of quasideterminant solutions.
\end{abstract}

2000 Mathematics Subject Classification. 35Q58, 46L55.

1. Introduction. Recently, there has been a lot of interest in non-commutative versions of some well-known soliton equations, such as the KP equation, the $\mathrm{KdV}$ equation, the Hirota-Miwa equation, the modified KP equation and the twodimensional Toda lattice $[\mathbf{1}, \mathbf{5}-\mathbf{9}, \mathbf{1 2}, \mathbf{1 4}, \mathbf{1 6}, \mathbf{1 8}, \mathbf{1 9}, \mathbf{2 1}-\mathbf{2 3}]$. There are a number of reasons for this lack of commutativity. For example, the variables might be square matrices or quarternions and so on. Another natural way in which the variables fail to commute is because of a quantization of the phase space resulting in the normal product being replaced by a Moyal product. In the approach taken here, it is not necessary to specify the reason for the lack of commutivity. Often, the non-commutative version is obtained simply by assuming that the coefficients in the Lax pair of the commutative equation do not commute.

Quasi-determinants [2-4] play the almost similar role in non-commutative algebra as determinants do in standard, commutative algebra. They arise in many situations where determinants appear in commutative algebra and this suggests that they are a very natural structure to use when working with non-commutative integrable systems. In particular, determinants are ubiquitous as solutions of commutative integrable systems and one way to obtain these is through the use of Darboux or binary Darboux transformations. Quasi-determinant solutions of non-commutative versions of these integrable systems arise in the same way. The reader is referred to the original papers [2-4] for a detailed and general treatment of quasi-determinants (see also [14] for a summary of the key results used in the current paper).

The semi-discrete Toda equation

$$
\frac{d}{d t} \log \left(\frac{v_{n}^{k+1}}{v_{n}^{k}}\right)=v_{n+1}^{k+1}+v_{n-1}^{k}-v_{n}^{k}-v_{n}^{k+1},
$$

was first considered in [11]. Some other related results were presented in $[\mathbf{1 3}, \mathbf{2 0}, \mathbf{2 4}]$. 
If one writes

$$
v_{n}^{k}=\frac{\tau_{n+1}^{k+1} \tau_{n-1}^{k}}{\tau_{n}^{k+1} \tau_{n}^{k}}
$$

and $\tau_{n}^{k}$ satisfies the Hirota bilinear equation

$$
D_{t} \tau_{n}^{k+1} \cdot \tau_{n}^{k}-\tau_{n+1}^{k+1} \tau_{n-1}^{k}+\tau_{n}^{k+1} \tau_{n}^{k}=0,
$$

then $v_{n}^{k}$ satisfies (1.1). In (1.2) $D_{t}$ denotes Hirota's bilinear operator defined by [10]

$$
\left.D_{t}^{n} a(t) \cdot b(t) \equiv\left(\frac{\partial}{\partial t}-\frac{\partial}{\partial t^{\prime}}\right)^{n} a(t) b\left(t^{\prime}\right)\right|_{t^{\prime}=t} .
$$

This paper is concerned with the following non-commutative generalization of (1.1):

$$
\begin{gathered}
v_{n}^{k} u_{n}^{k}=u_{n}^{k+1} v_{n+1}^{k}, \\
v_{n+1, t}^{k}+u_{n+1}^{k+1}-u_{n}^{k}=0,
\end{gathered}
$$

in which $u_{n}$ and $v_{n}$ and their derivatives do not commute in general. By introducing new variable $X_{n}^{k}$ where

$$
u_{n}^{k}=X_{n}^{k}\left(X_{n+1}^{k}\right)^{-1}, \quad v_{n}^{k}=X_{n}^{k+1}\left(X_{n}^{k}\right)^{-1},
$$

(1.3) is satisfied identically and (1.4) becomes

$$
\left(X_{n+1}^{k+1}\left(X_{n+1}^{k}\right)^{-1}\right)_{t}+X_{n+1}^{k+1}\left(X_{n+2}^{k+1}\right)^{-1}-X_{n}^{k}\left(X_{n+1}^{k}\right)^{-1}=0 .
$$

In the commutative reduction, it is easy to show, by writing $X_{n}^{k}=\tau_{n-1}^{k} / \tau_{n}^{k}$, that (1.5) becomes

$$
\begin{aligned}
& \tau_{n+1}^{k+1} \tau_{n+1}^{k}\left(D_{t} \tau_{n}^{k+1} \cdot \tau_{n}^{k}-\tau_{n+1}^{k+1} \tau_{n-1}^{k}+\tau_{n}^{k+1} \tau_{n}^{k}\right) \\
& \quad-\tau_{n}^{k} \tau_{n}^{k+1}\left(D_{t} \tau_{n+1}^{k+1} \cdot \tau_{n+1}^{k}-\tau_{n+2}^{k+1} \tau_{n}^{k}+\tau_{n+1}^{k+1} \tau_{n+1}^{k}\right)=0,
\end{aligned}
$$

which is satisfied whenever the bilinear semi-discrete Toda equation (1.2) is satisfied, thus verifying that indeed (1.3) and (1.4) is a non-commutative generalization of (1.1). For the rest of this paper, we will refer to (1.5) as the non-commutative semi-discrete Toda equation.

The main results of this paper are to show that this non-commutative system is integrable in the sense that it has a Lax pair and the associated Darboux and binary Darboux transformations may be iterated to construct families of exact solutions. We show how these solutions may be expressed in terms of quasi-determinants of two different types.

The paper is organized as follows. In Section 2, we present a Lax pair and its Darboux transformation and describe how iteration of this transformation gives quasicasoratian solutions. An adjoint linear problem and binary Darboux transformations are discussed in Section 3, and quasi-grammian solutions are obtained. Conclusions are given in Section 4. 
2. Quasi-casoratian solutions obtained by Darboux transformations. The noncommutativesemi-discrete Toda lattice (1.5) has Lax pair

$$
\begin{aligned}
\phi_{n, t}^{k} & =X_{n}^{k}\left(X_{n+1}^{k}\right)^{-1} \phi_{n+1}^{k}, \\
\phi_{n+1}^{k+1} & =\phi_{n}^{k}-X_{n+1}^{k+1}\left(X_{n+1}^{k}\right)^{-1} \phi_{n+1}^{k} .
\end{aligned}
$$

This was obtained by discretizing the Lax pair of the non-Abelian Toda lattice $[\mathbf{1 5}, \mathbf{1 7}]$ and simplifying the Lax pair of the semi-discrete Toda equation with self-consistent sources [20].

Let $\theta_{n, i}^{k}, i=1, \ldots, N$ be a particular set of eigenfunctions of the linear system and from these define the row vector $\Theta_{n}^{k}=\left(\theta_{n, 1}^{k}, \ldots, \theta_{n, N}^{k}\right)$. The Darboux transformation, determined by the particular solution $\theta_{n}^{k}$, for the non-commutative semi-discrete Toda lattice is

$$
\begin{aligned}
\widetilde{\phi}_{n}^{k} & =\phi_{n}^{k}-\theta_{n}^{k}\left(\theta_{n+1}^{k}\right)^{-1} \phi_{n+1}^{k}, \\
\widetilde{X}_{n}^{k} & =\theta_{n}^{k}\left(\theta_{n+1}^{k}\right)^{-1} X_{n+1}^{k} .
\end{aligned}
$$

This transformation may be iterated by defining

$$
\begin{aligned}
& \phi_{n}^{k}[l+1]=\phi_{n}^{k}[l]-\theta_{n}^{k}[l]\left(\theta_{n+1}^{k}[l]\right)^{-1} \phi_{n+1}^{k}[l], \\
& X_{n}^{k}[l+1]=\theta_{n}^{k}[l]\left(\theta_{n+1}^{k}[l]\right)^{-1} X_{n+1}^{k}[l],
\end{aligned}
$$

where $\phi_{n}^{k}[1]=\phi_{n}^{k}, X_{n}^{k}[1]=X_{n}^{k}$ and

$$
\theta_{n}^{k}[l]=\left.\phi_{n}^{k}[l]\right|_{\phi_{n}^{k} \rightarrow \theta_{n, l}^{k}}
$$

In particular,

$$
\begin{aligned}
& \phi_{n}^{k}[2]=\phi_{n}^{k}-\theta_{n, 1}^{k}\left(\theta_{n+1,1}^{k}\right)^{-1} \phi_{n+1}^{k}, \\
& X_{n}^{k}[2]=\theta_{n, 1}^{k}\left(\theta_{n+1,1}^{k}\right)^{-1} X_{n+1}^{k} .
\end{aligned}
$$

In what follows, we shall show by induction that the results of $N$ repeated Darboux transformations, $\phi_{n}^{k}[N+1]$ and $X_{n}^{k}[N+1]$, can be expressed in closed form as quasideterminants

$$
\phi_{n}^{k}[N+1]=\left|\begin{array}{cc}
\Theta_{n}^{k} & \phi_{n}^{k} \\
\Theta_{n+1}^{k} & \phi_{n+1}^{k} \\
\vdots & \vdots \\
\Theta_{n+N}^{k} & \phi_{n+N}^{k}
\end{array}\right|, \quad X_{n}^{k}[N+1]=(-1)^{N}\left|\begin{array}{cc}
\Theta_{n}^{k} & 0 \\
\Theta_{n+1}^{k} & 0 \\
\vdots & \vdots \\
\Theta_{n+N}^{k} & 1
\end{array}\right| X_{n+N}^{k}
$$

The initial case $N=1$ follows directly from (2.6) and (2.7). Also, using the noncommutative Jacobi identity, row homological relations and definition of quasi-Plücker 
coordinates (see (2.3)-(2.5) in [14] for example), we have

$$
\begin{aligned}
& \phi_{n}^{k}[N+2] \\
& =\phi_{n}^{k}[N+1]-\theta_{n}^{k}[N+1] \theta_{n+1}^{k}[N+1]^{-1} \phi_{n+1}^{k}[N+1]
\end{aligned}
$$

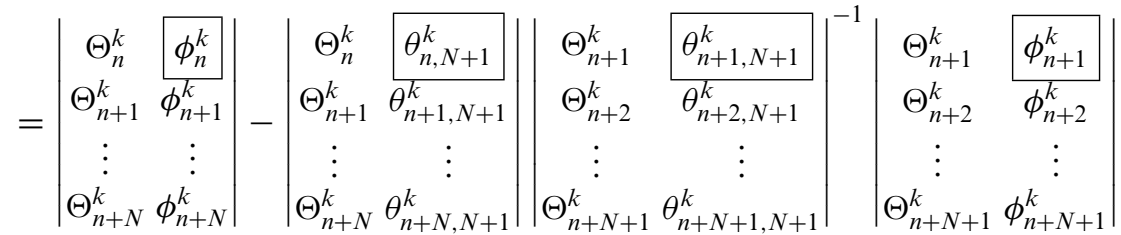

$$
\begin{aligned}
& =\left|\begin{array}{cc}
\Theta_{n}^{k} & \phi_{n}^{k} \\
\Theta_{n+1}^{k} & \phi_{n+1}^{k} \\
\vdots & \vdots \\
\Theta_{n+N}^{k} & \phi_{n+N}^{k}
\end{array}\right|-\left|\begin{array}{cc}
\Theta_{n}^{k} & \theta_{n, N+1}^{k} \\
\Theta_{n+1}^{k} & \theta_{n+1, N+1}^{k} \\
\vdots & \vdots \\
\Theta_{n+N}^{k} & \theta_{n+N, N+1}^{k}
\end{array}\right|\left|\begin{array}{cc}
\Theta_{n+1}^{k} & \theta_{n+1, N+1}^{k} \\
\Theta_{n+2}^{k} & \theta_{n+2, N+1}^{k} \\
\vdots & \vdots \\
\Theta_{n+N+1}^{k} & \theta_{n+N+1, N+1}^{k}
\end{array}\right|\left|\begin{array}{cc}
\Theta_{n+1}^{k} & \phi_{n+1}^{k} \\
\Theta_{n+2}^{k} & \phi_{n+2}^{k} \\
\vdots & \vdots \\
\Theta_{n+N+1}^{k} & \phi_{n+N+1}^{k}
\end{array}\right| \\
& =\left|\begin{array}{ccc}
\Theta_{n}^{k} & \theta_{n, N+1}^{k} & \phi_{n}^{k} \\
\Theta_{n+1}^{k} & \theta_{n+1, N+1}^{k} & \phi_{n+1}^{k} \\
\vdots & \vdots & \\
\Theta_{n+N+1}^{k} & \theta_{n+N+1, N+1}^{k} & \phi_{n+N+1}^{k}
\end{array}\right| .
\end{aligned}
$$

In a similar way, we also have

$$
\begin{aligned}
& X_{n}^{k}[N+2]=\theta_{n}^{k}[N+1] \theta_{n+1}^{k}[N+1]^{-1} X_{n+1}^{k}[N+1] \\
& =(-1)^{N}\left|\begin{array}{cc}
\Theta_{n}^{k} & \theta_{n, N+1}^{k} \\
\Theta_{n+1}^{k} & \theta_{n+1, N+1}^{k} \\
\vdots & \vdots \\
\Theta_{n+N}^{k} & \theta_{n+N, N+1}^{k}
\end{array}\right|\left|\begin{array}{cc}
\Theta_{n+1}^{k} & \theta_{n+1, N+1}^{k} \\
\Theta_{n+2}^{k} & \theta_{n+2, N+1}^{k} \\
\vdots & \vdots \\
\Theta_{n+N+1}^{k} & \theta_{n+N+1, N+1}^{k}
\end{array}\right| \begin{array}{ccc}
-1 & \Theta_{n+1}^{k} & 0 \\
\Theta_{n+2}^{k} & 0 \\
\vdots & \vdots \\
\Theta_{n+N+1}^{k} & 1
\end{array} \mid X_{n+N+1}^{k} \\
& =(-1)^{N}\left|\begin{array}{cc}
\Theta_{n}^{k} & \theta_{n, N+1}^{k} \\
\Theta_{n+1}^{k} & \theta_{n+1, N+1}^{k} \\
\vdots & \vdots \\
\Theta_{n+N}^{k} & \theta_{n+N, N+1}^{k}
\end{array}\right|\left|\begin{array}{cc}
\Theta_{n+1}^{k} & \theta_{n+1, N+1}^{k} \\
\vdots & \vdots \\
\Theta_{n+N}^{k} & \theta_{n+N, N+1}^{k} \\
\Theta_{n+N+1}^{k} & \theta_{n+N+1, N+1}^{k}
\end{array}\right| X_{n+N+1}^{k} \\
& =(-1)^{N+1}\left|\begin{array}{ccc}
\Theta_{n}^{k} & \theta_{n, N+1}^{k} & 0 \\
\Theta_{n+1}^{k} & \theta_{n+1, N+1}^{k} & 0 \\
\vdots & \vdots & \\
\Theta_{n+N+1}^{k} & \theta_{n+N+1, N+1}^{k} & 1
\end{array}\right| X_{n+N+1}^{k} .
\end{aligned}
$$

This proves the inductive step and the proof is complete. 
3. Quasi-grammian solutions obtained by binary Darboux transformations. The linear equations (2.1) and (2.2) have the formal adjoint

$$
\begin{aligned}
-\psi_{n, t}^{k} & =u_{n-1}^{k}{ }^{\dagger} \psi_{n-1}^{k}, \\
\psi_{n+1}^{k+1} & =\psi_{n}^{k}+v_{n}^{k^{\dagger}} \psi_{n}^{k+1} .
\end{aligned}
$$

As always with discrete Lax equations, some care is needed in defining their adjoint. The key point is that they should be chosen in such a way that the compatibility condition for (3.1) and (3.2) is identical to that of (2.1) and (2.2). In other words, (3.1) and (3.2) also form a Lax pair for (1.1).

Following the standard construction of a binary Darboux transformation, one introduces a potential $\Omega_{n}^{k}=\Omega\left(\phi_{n}^{k}, \psi_{n}^{k}\right)$ satisfying the following three conditions:

$$
\begin{gathered}
\Omega_{n, t}^{k}=-\psi_{n}^{k^{\dagger}} u_{n}^{k} \phi_{n+1}^{k}, \\
\Omega_{n}^{k+1}-\Omega_{n}^{k}=\psi_{n+1}^{k+1} \phi_{n}^{k}, \\
\Omega_{n+1}^{k}-\Omega_{n}^{k}=-\psi_{n+1}^{k} \phi_{n+1}^{k} .
\end{gathered}
$$

A binary Darboux transformation is then defined by

$$
\begin{aligned}
\phi_{n}^{k}[N+1] & =\phi_{n}^{k}[N]-\theta_{n}^{k}[N] \Omega\left(\theta_{n}^{k}[N], \rho_{n}^{k}[N]\right)^{-1} \Omega\left(\phi_{n}^{k}[N], \rho_{n}^{k}[N]\right), \\
\psi_{n}^{k}[N+1] & =\psi_{n}^{k}[N]-\rho_{n}^{k}[N] \Omega\left(\theta_{n-1}^{k}[N], \rho_{n-1}^{k}[N]\right)^{-\dagger} \Omega\left(\theta_{n-1}^{k}[N], \psi_{n-1}^{k}[N]\right)^{\dagger}, \\
X_{n}[N+1] & =\left(I+\theta_{n}^{k}[N] \Omega\left(\theta_{n}^{k}[N], \rho_{n}^{k}[N]\right)^{-1} \rho_{n}^{k}[N]^{\dagger}\right) X_{n}^{k}[N],
\end{aligned}
$$

where $\phi_{n}^{k}[1]=\phi_{n}^{k}, \psi_{n}^{k}[1]=\psi_{n}^{k}, X_{n}^{k}[1]=X_{n}^{k}$ and

$$
\theta_{n}^{k}[N]=\left.\phi_{n}^{k}[N]\right|_{\phi_{n}^{k} \rightarrow \theta_{n, N}^{k}}, \quad \rho_{n}^{k}[N]=\left.\psi_{n}^{k}[N]\right|_{\psi_{n}^{k} \rightarrow \rho_{n, N}^{k}} .
$$

Using the notation $\Theta_{n}^{k}=\left(\theta_{n, 1}^{k}, \ldots, \theta_{n, N}^{k}\right)$ and $P_{n}^{k}=\left(\rho_{n, 1}^{k}, \ldots, \rho_{n, N}^{k}\right)$, it is easy to prove by induction that for $N \geq 1$,

$$
\begin{aligned}
& \phi_{n}^{k}[N+1]=\left|\begin{array}{cc}
\Omega\left(\Theta_{n}^{k}, P_{n}^{k}\right) & \Omega\left(\phi_{n}^{k}, P_{n}^{k}\right) \\
\Theta_{n}^{k} & \phi_{n}^{k}
\end{array}\right|, \\
& \psi_{n}^{k}[N+1]=\left|\begin{array}{cc}
\Omega\left(\Theta_{n-1}^{k}, P_{n-1}^{k}\right)^{\dagger} & \Omega\left(\Theta_{n-1}^{k}, \psi_{n-1}^{k}\right)^{\dagger} \\
P_{n}^{k} & \psi_{n}^{k}
\end{array}\right|
\end{aligned}
$$

and

$$
\Omega\left(\phi_{n}^{k}[N+1], \psi_{n}^{k}[N+1]\right)=\left|\begin{array}{ll}
\Omega\left(\Theta_{n}^{k}, P_{n}^{k}\right) & \Omega\left(\phi_{n}^{k}, P_{n}^{k}\right) \\
\Omega\left(\Theta_{n}^{k}, \psi_{n}^{k}\right) & \Omega\left(\phi_{n}^{k}, \psi_{n}^{k}\right)
\end{array}\right| .
$$

We may thus after $N$ binary Darboux transformations obtain

$$
X_{n}^{k}[N+1]=-\left|\begin{array}{cc}
\Omega\left(\Theta_{n}^{k}, P_{n}^{k}\right) & P_{n}^{k^{\dagger}} \\
\Theta_{n}^{k} & -I
\end{array}\right| X_{n}^{k}
$$


In fact, we can prove the above results by induction. Using (3.10)-(3.12), we have

$$
\begin{aligned}
& X_{n}^{k}[N+2]=\left(I+\Theta_{n}^{k}[N+1] \Omega\left(\Theta_{n}^{k}[N+1], P_{n}^{k}[N+1]\right)^{-1} P_{n}^{k}[N+1]^{\dagger}\right) X_{n}^{k}[N+1] \\
& =-\left(I+\left|\begin{array}{cr}
\Omega\left(\Theta_{n}^{k}, P_{n}^{k}\right) & \Omega\left(\theta_{n, N+1}^{k}, P_{n}^{k}\right) \\
\Theta_{n}^{k} & \theta_{n, N+1}^{k}
\end{array}\right|\left|\begin{array}{cc}
\Omega\left(\Theta_{n}^{k}, P_{n}^{k}\right) & \Omega\left(\theta_{n, N+1}^{k}, P_{n}^{k}\right) \\
\Omega\left(\Theta_{n}^{k}, \rho_{n, N+1}^{k}\right) & \Omega\left(\theta_{n, N+1}^{k}, \rho_{n, N+1}^{k}\right)
\end{array}\right|^{-1}\right. \\
& \left.\left|\begin{array}{cc}
\Omega\left(\Theta_{n-1}^{k}, P_{n-1}^{k}\right) & P_{n}^{k^{\dagger}} \\
\Omega\left(\Theta_{n-1}^{k}, \rho_{n-1, N+1}^{k}\right) & \rho_{n, N+1}^{k}{ }^{\dagger}
\end{array}\right|\right)\left|\begin{array}{cc}
\Omega\left(\Theta_{n}^{k}, P_{n}^{k}\right) & P_{n}^{k^{\dagger}} \\
\Theta_{n}^{k} & -I
\end{array}\right| X_{n}^{k} .
\end{aligned}
$$

Noticing that

$$
\begin{aligned}
& \left|\begin{array}{cc}
\Omega\left(\Theta_{n-1}^{k}, P_{n-1}^{k}\right) & P_{n}^{k^{\dagger}} \\
\Omega\left(\Theta_{n-1}^{k}, \rho_{n-1, N+1}^{k}\right) & \rho_{n, N+1}^{k}{ }^{\dagger}
\end{array}\right| \begin{array}{cc}
\Omega\left(\Theta_{n}^{k}, P_{n}^{k}\right) & P_{n}^{k^{\dagger}} \\
\Theta_{n} & -I
\end{array} \mid \\
& =-\left(\rho_{n, N+1}^{k}{ }^{\dagger}-\Omega\left(\Theta_{n-1}^{k}, \rho_{n-1, N+1}^{k}\right) \Omega\left(\Theta_{n-1}^{k}, P_{n-1}^{k}\right)^{-1} P_{n}^{k^{\dagger}}\right)\left(I+\Theta_{n}^{k} \Omega\left(\Theta_{n}^{k}, P_{n}^{k}\right)^{-1} P_{n}^{k^{\dagger}}\right) \\
& =-\rho_{n, N+1}^{k}+\Omega\left(\Theta_{n-1}^{k}, \rho_{n-1, N+1}^{k}\right) \Omega\left(\Theta_{n-1}^{k}, P_{n-1}^{k}\right)^{-1} P_{n}^{k^{\dagger}} \\
& +\left(\Omega\left(\Theta_{n}^{k}, \rho_{n, N+1}^{k}\right)-\Omega\left(\Theta_{n-1}^{k}, \rho_{n-1, N+1}^{k}\right)\right) \Omega\left(\Theta_{n}^{k}, P_{n}^{k}\right)^{-1} P_{n}^{k^{\dagger}} \\
& +\Omega\left(\Theta_{n-1}^{k}, \rho_{n-1, N+1}^{k}\right) \Omega\left(\Theta_{n-1}^{k}, P_{n-1}^{k}\right)^{-1}\left(\Omega\left(\Theta_{n-1}^{k}, P_{n-1}^{k}\right)-\Omega\left(\Theta_{n}^{k}, P_{n}^{k}\right)\right) \Omega\left(\Theta_{n}^{k}, P_{n}^{k}\right)^{-1} P_{n}^{k^{\dagger}} \\
& =-\rho_{n, N+1}^{k}+\Omega\left(\Theta_{n}^{k}, \rho_{n, N+1}^{k}\right) \Omega\left(\Theta_{n}^{k}, P_{n}^{k}\right)^{-1} P_{n}^{k^{\dagger}} \\
& =-\left|\begin{array}{cc}
\Omega\left(\Theta_{n}^{k}, P_{n}^{k}\right) & P_{n}^{k^{\dagger}} \\
\Omega\left(\Theta_{n}^{k}, \rho_{n, N+1}^{k}\right) & \left.\rho_{n, N+1}^{k}\right|^{\dagger}
\end{array}\right|,
\end{aligned}
$$

it follows that

$$
X_{n}^{k}[N+2]=-\left|\begin{array}{ccc}
\Omega\left(\Theta_{n}^{k}, P_{n}^{k}\right) & \Omega\left(\theta_{n, N+1}^{k}, P_{n}^{k}\right) & P_{n}^{k^{\dagger}} \\
\Omega\left(\Theta_{n}^{k}, \rho_{n, N+1}^{k}\right) & \Omega\left(\theta_{n, N+1}^{k}, \rho_{n, N+1}^{k}\right) & \rho_{n, N+1}^{k} \\
\Theta_{n}^{k} & \theta_{n, N+1}^{k} & -I
\end{array}\right| X_{n}^{k},
$$

as required.

4. Conclusions. In this paper, we have described a non-commutative version of the semi-discrete Toda equation. We have obtained quasi-casoratian and quasigrammian solutions by means of discrete Darboux transformations and binary Darboux transformations, respectively. We have given an inductive proof of the iterated Darboux transformations and solutions by using quasi-determinant identities. Since we have not at any point specified the nature of non-commutativity, the results obtained here are valid whatever the reason for non-commutativity is.

ACKnOwledgements. This work was supported by the Royal Society China Fellowship and the National Natural Science Foundation of China (grant no. 10601028). 


\section{REFERENCES}

1. A. Dimakis and F. Müller-Hoissen, An algebraic scheme associated with the noncommutative KP hierarchy and some of its extensions, J. Phys. A 38 (2005), 5453-5505.

2. P. Etingof, I. M. Gelfand and V. S. Retakh, Nonabelian integrable systems, quasideterminants, and Marchenko lemma, Math. Res. Lett. 5 (1998), 1-12.

3. I. M. Gelfand, S. Gelfand, V. S. Retakh and R. L. Wilson, Quasideterminants, $A d v$. Math. 193 (2005), 56-141.

4. I. M. Gelfand and V. S. Retakh, Determinants of matrices over noncommutative rings, Funct. Anal. Prilozhen. 25 (1991) 13-25.

5. C. R. Gilson and J. J. C. Nimmo, On a direct approach to quasideterminant solutions of a noncommutative KP equation, J. Phys. A 40 (2007), 3839-3850.

6. C. R. Gilson, J. J. C. Nimmo and Y. Ohta, Quasideterminant solutions of a non-Abelian Hirota-Miwa equation, J. Phys. A: Math. Theor. 40 (2007), 12607-12617.

7. C. R. Gilson, J. J. C. Nimmo and C. M. Sooman, On a direct approach to quasideterminant solutions of a noncommutative modified KP equation, J. Phys. A: Math. Theor. 41 (2008), 085202 (10 pp.).

8. M. Hamanaka and K. Toda, Towards noncommutative integrable systems, Phys. Lett. A 316 (2003), 77-83.

9. M. Hamanaka, Noncommutative solitons and D-branes, PhD Thesis (Nagoya University, Japan). arXiv:hep-th/0303256 (2003).

10. R. Hirota, The direct method in soliton theory (A. Nagai, J. J. C. Nimmo and C. R. Gilson, Editors) (Cambridge University Press, Cambridge, UK, vol. 155, 2004).

11. R. Inoue and K. Hikami, Construction of soliton cellular automaton from the vertex model - the discrete 2D Toda equation and the Bogoyavlensky lattice, J. Phys. A: Math. Gen. 32 (1999), 6853-6868.

12. B. A. Kupershmidt, $K P$ or $m K P$ : Noncommutative mathematics of Lagrangian, Hamiltonian, and integrable systems. Mathematical Surverys and Monographs (American Mathematical Society, New York, 78, 2000).

13. C. X. Li and X. B. Hu, Pfaffianization of the semi-discrete Toda equation, Phys. Lett. A 329 (2004), 193-198.

14. C. X. Li and J. J. C. Nimmo, Quasideterminant solutions of a non-Abelian Toda lattice and kink solutions of a matrix sine-Gordon equation, Proc. R. Soc. A 464 (2008), 951-966.

15. A. V. Mikhailov, Integrability of a two-dimensional generalization of the Toda chain, JETP Lett. 30 (1979), 443-448. 5065 .

16. J. J. C. Nimmo, On a non-Abelian Hirota-Miwa equation, J. Phys. A 39 (2006), $5053-$

17. J. J. C. Nimmo and R. Willox, Darboux transformations for the two-dimensional Toda system, Proc. R. Soc. London Ser. A 453 (1997), 2497-2525.

18. L. D. Paniak, Exact noncommutative KP and KdV multi-solitons, arXiv:hepth/0105185 (2001).

19. M. Sakakibara, Factorization methods for noncommutative KP and Toda hierarchy, J. Phys. A 37 (2004), L599-L604.

20. H. Y. Wang, Integrability of the semi-discrete Toda equation with self-consistent sources, J. Math. Anal. Appl. 330 (2007), 1128-1138.

21. N. Wang and M. Wadati, Noncommutative extension of $\bar{\partial}$-dressing method, J. Phys. Soc. Jpn. 72 (2003), 1366-1373.

22. N. Wang and M. Wadati, Exact Multi-line Soliton Solutions of Noncommutative KP Equation, J. Phys. Soc. Jpn. 72 (2003), 1881-1888.

23. N. Wang and M. Wadati, Noncommutative KP hierarchy and Hirota triple-product relations, J. Phys. Soc. Jpn. 73 (2004), 1689-1698.

24. J. X. Zhao, Commutativity of Pfaffianization and Bäcklund transformation: The semidiscrete Toda equation, Math. Comput. Simul. 74 (2007), 388-396. 